\title{
Built-In-Test Capability Evaluation for Weapon Systems Using Computer Modeling
}

\author{
Fred Iiguori * Naval Air Warfare Center * Lakehurst
}

Key Words: Weapon system modeling, built-in-test, testability, testability evaluation

\section{SUMMARY \& CONCLUSIONS}

A critical concern in designing or modifying a weapon system is the ability to support that system throughout its life cycle. Supportability begins with the ability to monitor the health of the system concurrently with its normal operation. It is common practice to include a built-in-test (BIT) capability that not only continuously monitors system health but also provides the first level of fault isolation. Evaluating the effectiveness of a built-in-test capability is a difficult task during the weapon system design phase because there is no effective way to test a "paper design".

The objective of this project is to develop a computer-aided tool to assist in the evaluation of a proposed BIT design and other attributes of a weapon system that influence supportability. Hence timely recommendations can be made for improving the design as well as providing critical information for planning the logistics support requirements for the weapon system.

The first approach was to build a hierarchical simulator that could synthesize a model of the weapon system by linking lower level models of the weapon system components. The model would contain weapon system attributes that impact testability and therefore supportability. An analysis of the model would yield the desired evaluation.
The first approach was "bottom up" model building attempt. It proved impractical because component models developed by existing computer-aided-design systems are not mutually compatible and do not contain fault mode information.

A second approach has been initiated by building the weapon system model "top down". That is, starting with a system model that was developed for other purposes but having the flexibility to be modified or expanded to encompass testability and supportability attributes. A number of existing modeling systems have been investigated and one has been identified that offers promise in being adaptable to the evaluation of weapon system testability and supportability.

\section{INTRODUCTION}

The complexity of each new weapon system increases dramatically. Multi-mission operational requirements continue to push new designs to the edge of current technology. While this philosophy of ever increasing sophistication has kept the United states ahead of other nations in weapon system capability, it has placed a severe stress on the ability to provide organic support. Continued dependence on a large inventory of field test equipment and highly trained maintenance personnel is becoming increasingly impractical. The 
weapon system itself must include a much greater capability to monitor its own health and identify failed subsystems or components. This is the role of the built-in-test (BIT) system. Some repairs can automatically be accomplished by switching to redundant components or switching to a back up mode of operation. U1timately, however, repairs will be required so those failures that are not self healing must be conveyed to the maintenance personnel for off line repair. The information needed to effect a rapid repair should also be provided by the BIT system.

The only way a weapon system can perform an effective selfdiagnosis is to have a comprehensive BIT system incorporated into the weapon system design beginning with its conceptual design and pursued throughout the design and development phase. Experience indicates that 80 percent or more of the opportunity to influence a design is lost after the initial conceptual design. The traditional methods of doing a supportability analysis during the weapon system development phase will never result in an effectivesupportability posture for modern weapon systems. Tools and processes must be developed that enable logisticians to evaluate weapons system designs for supportability concurrently with the system design and development process. The objective of this project is to develop such tools and procedures. The key requirement is the ability to model a weapon system with sufficient granularity that testability and supportability attributes can be represented and evaluated.

\section{CURRENT STATUS OF MODELING}

Some computer aids already exist for assisting in logistic analysis of weapon systems.
Programs exist for computing mean-time-between-failure (MBTF) and for performing reliability analyses. Such programs aid in performing complex calculations using data collected manually in a labor intensive and error prone process. Computer aided design systems are much more sophisticated and are potentially useful for supportability analysis but they have two shortcomings: (1) they tend to be unique designs not format or structurally compatible with each other and (2) the do not contain failure mode data on the products that they model. These shortcomings preclude building higher level models from lower models or evaluating designs for test-ability.

System level models have been developed for a wide variety of applications. These however are narrowly designed to evaluate very specific attributes of a proposed design and do not represent the system by a generic model useful for a multiplicity of analyses such as would be required for testability or supportability analyses. A number of system modeling tools have been investigated but none have been found to date that represent a system with sufficient definition as to be useful for supportability analyses. Recently some simulators have been designed to model systems using "frame" designs that lend themselves to hosting software modules designed by the user. Thus specific attributes of a system can be modeled by a user without having to design a unique simulator from scratch. It is believed that such a simulator could be adapted to solving the supportability analysis problem.

\section{WEAPON SYSTEM MODELING}

\subsection{Supportability Defined}


The official Department of Defense definition o supportability is given in the military standard MIL-STD-1388-1A as follows:

"The degree to which system design characteristics and planned logistics resources, including manpower, meet system peacetime operational and wartime utilization requirements."

It is further explained that supportability is governed by the characteristics of the entire support system that facilitate the maintenance of the prime equipment. The support system consists of all assets involved in supporting a piece of equipment or a weapon system.

As defined in MIL-STD-1388-1A, there are four distinctly different parts to supportability, namely: attributes

* Equipment or system design

* Logistics support system assets (spares, manuals, etc.)

* Peacetime objectives (cost effectiveness)

* Wartime objectives (surge capability)

of these the driving function that impacts all aspects of supportability is the system design. If the design does not provide for testability and repairability little can be done after the fact to make the system an effectively supported product. And since $80 \%$ of the system's supportability attributes are fixed by during the conceptual design process, it is critical that concerns about supportability be addressed at that time.

3.2 Design Attributes Impacting Supportability

Design attributes impacting supportability are the intrinsic characteristics of the prime equipment that require infrequent maintenance and ensure ease of maintenance. The most critical characteristics are: reliability, maintainability, test equipment compatibility, test point access, and BIT capability. Figure 1 illustrates these five characteristics and identifies some of the subordinate attributes of each. BIT is the key to health assessment and the starting point of the troubleshooting and maintenance process. If it is inadequate or misleading, the maintenance task is greatly impaired or prolonged. Hence the first concern of a supportability analysis is to evaluate the effectiveness of the BIT system.

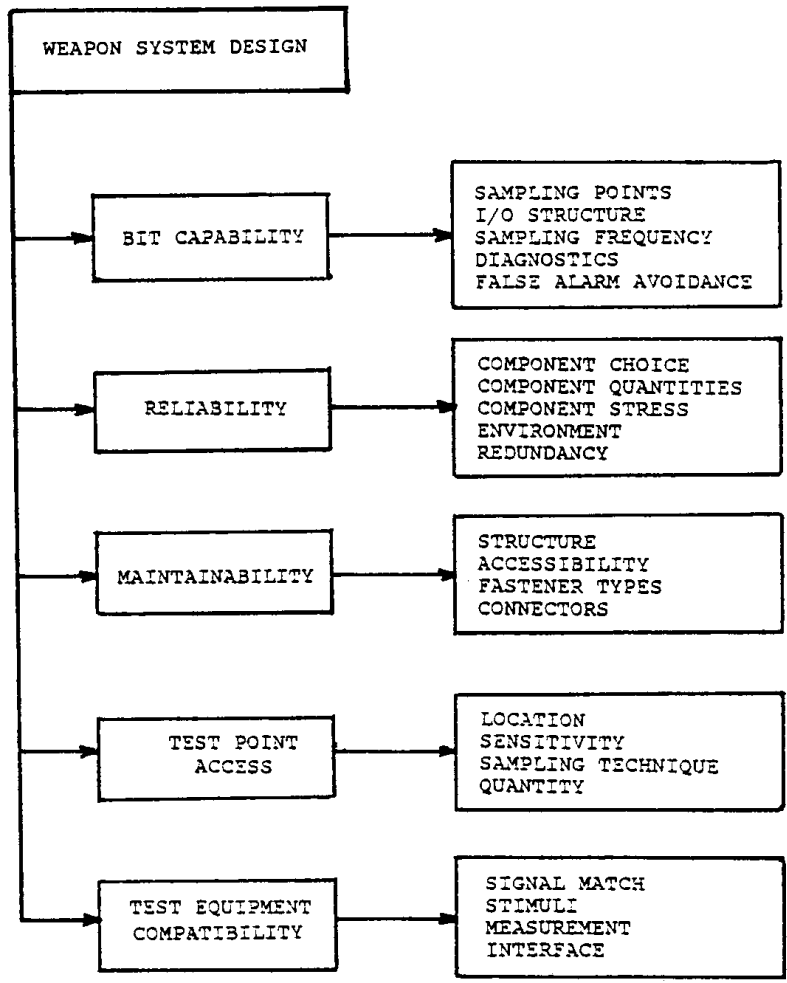

Figure 1 - Design Attributes That Determine Supportability

\subsection{Weapon System Modeling}

The weapon system model is constructed using an existing software package developed by Duke 
University under NASA sponsorship that is in the public domain. The system is called HARP, an acronym for Hybrid Automated Reliability Predictor. Although it was designed to implement advanced reliability modeling techniques it is modularly constructed so as to facilitate adaptation to other modeling problems. HARP is described in Reference (1).

HARP is designed to perform system level modeling. A system level analysis is performed by separately analyzing each subsystem and combining the results to obtain the final solution. This is referred to as structural decom- position. This technique lends itself well to weapon system analysis. The weapon system model can then be constructed similarly to its "family tree" or its structural breakdown from system to subsystem to major to $\mathrm{com}-$ ponents.

\subsection{BIT Analysis System Require-} ments

The required elements of a BIT analysis system can be defined in terms of the classical work breakdown structure. Figure 1 represents the first level of the work breakdown wherein the principal elements of supportability

Table 1 - Software Modules Comprising the BIT Analysis System

(a) Design for testability - This is the process of determining the test access method that should be incorporated in the product design to assure that it can be adequately tested with little or no manual intervention. Design techniques and software tools exist for digital electronic assemblies but remain to be developed for analog electronic and nonelectronic assemblies.

(b) Test point selection - This is the process of selecting which test points are to be incorporated in a given design. Software exists for assisting the designer in this process which can be readily incorporated in the BIT evaluator.

(c) Sampling effectiveness Equally important to selecting effective test points is the the need to determine the method and frequency for sampling the signals at the test points. This is a systems analysis process requiring highly skilled systems engineers. It remains labor intensive with no single tool to solve the problem. (d) Diagnostic analysis - some programs exist but have only been applied at the subassembly and assembly level of a weapon system. The algorithm should be extendible to handle system level diagnostics. HARP has a powerful fault tree diagnostic module that should prove effective in this process.

(e) Ambiguity group analysis - Unambiguous identification of a failed component or assembly is not always possible so a method of identifying ambiguity groups is required. This can be accomplished as an extension of the diagnostic fault tree analyzer.

(f) Test parameter evaluation This is the process of determining if the compliment of test points accessed by the BIT system are adequate to provide effective health monitoring and required level of fault isolation without manual intervention. This is the key to BIT system evaluation and the most difficult part of the process. No computer-aided tools exist to help in this process. It remains the principal challenge of the project. 
are defined. The second level of the work breakdown identifies the tools and processes required to evaluate a BIT system. These are provided in the form of software modules that comprise the BIT evaluation system. Some of these modules have already been developed and some remain to be designed and tested. Table 1 provides a breakdown of the required modules and the current status of their development.

\subsection{Attribute Table Development}

A methodology must be developed to determine and specify parameters that must be monitored by an effective BIT system. The approach to be used is to develop individual attribute tables for each subsystem, major assembly and subassembly that is monitored by the BIT system. Developing these tables requires an intimate understanding of the function and design of each item to be monitored by the system. The format and structure can be provided by a software tool but the quantitative information must be generated manually since CAD/CAM systems of today do not provide the parametric information required. In the future this may become a by-product of the design process.

\subsection{BIT Evaluation System Design}

A conceptual design of the BIT evaluation system is shown in Figure 2. The HARP system provides the frame for the system as well as the fault tree analysis process. The attribute tables required by the system have been described. The various software tools are described in Table 1 . The BIT analysis ariver is the software module that controls the evaluation process and represents an original design effort. The output of the evaluation process is a BIT effectiveness report.

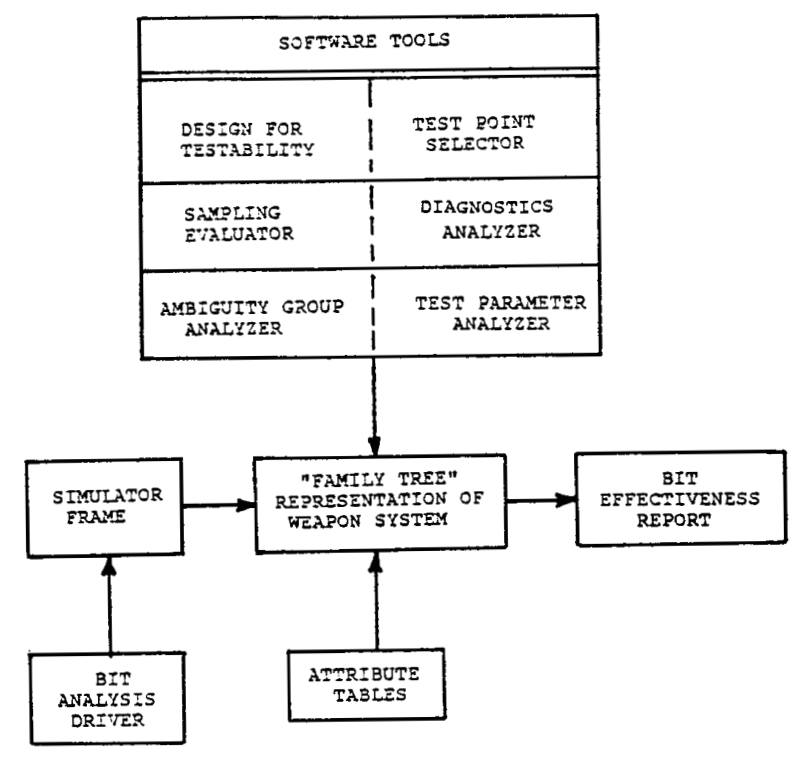

Figure 2 - Conceptual Design of the BIT Evaluation system

\section{REFERENCES :}

1. MIL-STD-1388-1A Logistic Support Analysis, Department of Defense, 11 April 1983.

2. S.J. Bavuso, et. al., "Application of the Hybrid Automated Reliability Predictor", NASA Technical Paper 2760, Dec. 1988.

3. S. Goldstein, et. al.,"Product Supportability Issues In Early Design Phases", Institute For Defense Analysis Paper P-2150, October 1989.

4. K. S. Trivedi, "HARP Workshop" Manual, Duke University Department of Computer Science, July 1990.

5. F. Liguori, "System Simulation for Supportability Analysis", IEEE

Built In self Test Workshop, Chariston, SC, Mar.21-23, 1990. 
6. F. Liguori, "Readiness Assessment - Where Ilities and Abilities Meet", Tri- Service RAMCAD Group Meeting, Rosslyn, VA, April 26, 1990.

7. F. Liguori, "Computer Modeling for system Level supportability Analyses", SOLE 25th Annual Logistics Symposium, Phoenix, AZ, Aug. 21-23, 1990 .

8. F. Liguori, "Weapon System Functional Modeling workstation, End of Year Report", Naval Air Warfare Center, Lakehurst, NJ, sep. $30,1991$.
Fred Liguori, PE

Naval Air Warfare Center, Aircraft Div.

Lakehurst, NJ 08733-5000 USA

Fred Iiguori is a senior level Technologist currently serving as the center's Technology Assessment Oficer. He is responsible for evaluating new scientific and technological advances and predicting their potential impacts on weapon system supportability. He is also the principal investigator for the supportability evaluation program. prior to this assignment he organized and managed the Automatic Test Equipment Branch which pioneered development and fleet introduction of early ATE deployed on aircraft carriers. His industrial experience includes management of the Software Systems Engineering Laboratory at Emerson Electric Company and as project engineer at RCA and spery. He is a Fellow De the IEE and a Kember of SOLE He has had more than 30 papers than 30 papers published in the field of ATE and weapon system support and edited the first published book on ATE. He has held held numerous leadership positions in the IEEE including AUTOTESTCON Chairman in 1972 and 1989 , Editor the Transactions on Instrumentations and Measurement, and president of the IEEE Instrumentation and Measurement society. He received an IEEE Distinguished service Award in 1984. He holds a BSEE from Tufts University and an MBA from Hofstra U. 